\title{
RECUPERACIÓN DE SOLVENTES DE UNA EMPRESA DEL SECTOR FLEXOGRÁFICO
}

\author{
RECOVERY OF SOLVENTS FROM A COMPANY IN THE FLEXOGRAPHIC SECTOR
}

Felipe Correa Mahecha*
María Alejandra Tamayo León**
Lizeth Catherine Arias Arias***

Recibido: 9 de junio de 2017
Aceptado: 4 de septiembre de 2017

\section{Resumen}

En este trabajo se realizó el muestreo y caracterización de los residuos líquidos de impresión generados por una empresa flexográfica. La investigación empleó el método descrito por la NTC 619 para determinar la curva de destilación y establecer la composición del destilado de nueve fracciones; posteriormente, usando un modelo de destilación diferencial con arreglo en diferencias finitas, se calcularon las composiciones instantáneas de fondos. Con el uso del programa Aspen Plus, se calcularon las temperaturas de burbuja bajo dos modelos termodinámicos, en los que se encontró diferencias significativas con los datos experimentales. También se elaboraron los mapas de curvas residuales para las composiciones normalizadas de los tres compuestos orgánicos volátiles mayoritarios de la mezcla, lo que reportó una buena predicción de la composición de fondos con los modelos UNIQUAC y NRTL, frente a los datos obtenidos experimentalmente.

Palabras clave: destilación diferencial, compuestos orgánicos volátiles, curva de destilación, residuos de tinta.

\section{Abstract}

In this work, the sampling and characterization of liquid print residues generated by a flexographic company were carried out. The research used the method described by the NTC 619 to determine the distillation curve and to establish the composition of the distillate of nine fractions; subsequently, using a differential distillation model with the arrangement of finite differences, the instantaneous structures of bottoms were calculated. With the use of the Aspen Plus program, it calculated the bubble temperatures under two thermodynamic models, in which it found significant differences with the experimental data. It also elaborated the residual curve maps for the standard compositions of the three-major volatile organic compounds of the mixture, which reported a right prediction of the structure of bottoms with the UNIQUAC and NRTL models, against the data obtained experimentally.

Keywords: differential distillation, volatile organic compounds, distillation curve, waste of ink.

\footnotetext{
* Ingeniero Químico, Docente investigador, Fundación Universidad de América, Bogotá, Colombia. ORCID:http:// orcid.org/0000-0003-1050-8222. felipe.correa@profesores.uamerica.edu.do

** Estudiante de Ingeniería química, Coinvestigadora, Fundación Universidad de América, Bogotá, Colombia. maria.tamayo@estudiantes.uamerica.edu.co

${ }^{* * *}$ Estudiante de Ingeniería química, Coinvestigadora, Fundación Universidad de América, Bogotá, Colombia. lizeth.arias@estudiantes.uamerica.edu.co
} 


\section{INTRODUCCIÓN}

La industria de impresión actualmente se encuentra en pleno crecimiento; no obstante, genera residuos peligros que contienen compuestos orgánicos volátiles, pigmentos y resinas, producto del lavado de planchas y equipos de impresión. A escala mundial, se calcula que cientos de miles de toneladas de estos residuos son anualmente incinerados o dispuestos en vertederos (Yan, Chen, Zhu y Yao, 2013).

En Colombia, la industria gráfica representó el 1.74 \% de la facturación del sector manufacturero durante el 2016, reportando ingresos operacionales de 2.98 billones de pesos (Andigraf, 2017). Por su parte, en el primer trimestre del 2017, la industria tuvo una participación del $3.5 \%$ del producto interno bruto (Ministerio de Industria y Turismo, 2017).

Estudios realizados en el 2014 muestran que el $7 \%$ de la tinta empleada en el proceso de impresión se convierte en residuo, y tan solo el $8 \%$ de los residuos líquidos peligrosos son recuperados o valorizados por las organizaciones (Andigraf, 2014). Se estima que durante el año 2015 se generaron 5910 toneladas de residuos peligrosos, producto de la fabricación y uso de tintas, colorantes, pigmentos, lacas y barnices (IDEAM, 2016).

En la actualidad, el mercado demanda productos impresos con un bajo impacto ambiental, por lo que se requiere, entre otros aspectos, la reducción, reutilización y reciclaje de los residuos que se producen durante el proceso de impresión. También se ha avanzado en el desarrollo de tintas más sostenibles con una baja huella ecológica, que se soporten en tecnologías con base acuosa y curado ultravioleta y usen recursos renovables; sin embargo, las formulaciones basadas en estas tecnologías son escasas (Robert, 2015), razón por la que cobra más relevancia la investigación en procesos de recuperación de solventes, pigmentos y otros subproductos derivados de los residuos generados por esta industria.

La destilación batch es la operación más empleada para la recuperación de solventes, debido a su eficacia y flexibilidad, toda vez que la composición y cantidad de la mezcla a recuperar puede variar con el tiempo (Smallwood, 2002). Esta destilación también proporciona soluciones de separación en una sola unidad y permite la separación de solventes en residuos con altos contenidos de sólidos y resinas (Sorensen, 2014)

Se han propuesto metodologías para la recuperación de solventes a partir de residuos de la industria gráfica, que incluyen la destilación azeotrópica (Yan et ál., 2013), así como metodologías para la recuperación de pigmentos que quedan en los fondos de destilación luego de la recuperación de los solventes (Pineda, Fullana y Aracid, 2011).

E1 presente trabajo desarrolla una metodología basada en pruebas de destilación, siguiendo la NTC 319. Método de ensayo para el intervalo de destilación de líquidos orgánicos volátiles (ICONTEC, 1995), en la que se obtienen nueve fracciones de destilado con los correspondientes intervalos de temperaturas experimentales, se realizó una cuantificación por cromatografía de gases de las fracciones obtenidas, posteriormente se usó un modelo de diferencias finitas con el fin de calcular las concentraciones en los fondos durante el proceso, finalmente se empleó la información experimental y la obtenida con el modelo para simular el proceso de destilación con el uso del programa Aspen Plus, obteniendo las curvas residuales para dos modelos termodinámicos, las que pueden ser usadas para el diseño y operación de los sistemas de destilación.

\section{Materiales y mÉtodos}

Las muestras de residuos de tinta fueron suministradas por una empresa de impresión flexográfica. Las cromatografías de gases acoplada a espectro de masas, la determinación de humedad, de sólidos no volátiles y la prueba de destilación fueron realizadas en el Centro de Gestión 
Industrial del Sena, seccional Bogotá. Las cromatografías de gases de las fracciones obtenidas en la prueba de destilación fueron ejecutadas por la empresa Tintas S. A.

\section{Materiales}

Se tomaron 40 litros de residuos de tinta producto del proceso de impresión y limpieza de los equipos de una industria de impresión flexográfica ubicada en la zona franca de Fontibón, con el fin de garantizar la representatividad y calidad de las muestras, y se siguieron los lineamientos de recolección, almacenamiento y preservación de la Norma Técnica Colombina 1052 (Icontec, 2008).

Las muestras fueron etiquetadas y almacenadas usando recipientes herméticos de polietileno de alta densidad, bajo condiciones adecuadas de luz, humedad y temperatura.

\section{Caracterización del residuo de tinta}

La humedad de las muestras fue determinada con un titulador automático V20 Metler Toledo ${ }^{\circledR}$ mediante el método de Karl Fischer; para ello, se estandarizó el reactivo Hidranal por triplicado utilizando agua tipo I como estándar al $100 \%$. El análisis de la muestra se realizó por pesada por diferencia en el vaso de valoración el reactivo Karl Fisher, siguiendo los procedimientos establecidos para la determinación de humedad en materiales de referencia (Rivera y Pérez, 2006).

El contenido de sólidos no volátiles presentes en las muestras se estableció mediante un análisis gravimétrico, siguiendo los protocolos señalados por NTC 762 (Icontec, 1974). $100 \mathrm{~mL}$ de muestra fueron pesados en un crisol previamente secado y tarado, para ser sometidos a un proceso de evaporación en estufa a $105^{\circ} \mathrm{C}$. Luego de media hora, la muestra fue enfriada en un desecador, pesada y devuelta al horno por un periodo de 15 minutos adicionales; después de este lapso, fue nuevamente enfriada en desecador y pesada con una balanza analítica. Este procedimiento se repitió hasta que dos pesadas consecutivas no difirieron en más de $0.1 \mathrm{mg}$. La masa seca fue determinada por diferencia con la masa original sustrayendo el peso del crisol.

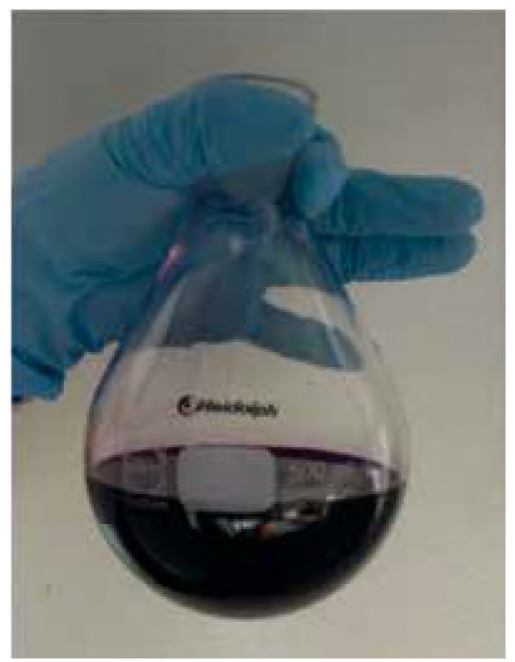

Figura 1. Muestra del residuo procesada.

En el Centro de Gestión Industrial del SENA, regional Bogotá, se realizó una cromatografía de gases acoplada a espectro de masas; el cromatógrafo operó con una columna SH 20(wax) capilar de sílica fusionada. Como fase estacionaria se empleó polietilenglicol en fase polar y como fase móvil se utilizó el He. $10 \mu \mathrm{L}$ de la muestra fueron tomados con una micropipeta y llevados a un 
volumen de $10 \mathrm{~mL}$ con ciclohexano. Finalmente, la muestra fue pasada por un filtro de nylon de 0.45 micrómetros, con el fin de remover los sólidos no solubles; luego fue trasferida a un vial de cromatografía para su análisis. Las condiciones de operación de la cromatografía de gases acoplada a espectro de masas (CG-EM) se presentan en la tabla 1.

Tabla 1. Condiciones de operación CG y CG-EM

\begin{tabular}{lll}
\hline \multicolumn{1}{c}{ Condición } & \multicolumn{1}{c}{ Cromatografía de gases (CG) } & \multicolumn{1}{c}{$\begin{array}{c}\text { Cromatografía de gases acoplada a } \\
\text { espectrómetro de masas (CG-EM) }\end{array}$} \\
\hline Tipo de columna. & Perkin Elmer Autosystem XL PE-5 & MS GCMS-QP $2010^{\mathrm{b}}$ \\
Fase móvil & $\mathrm{H}_{2}$, aire, He & Helio \\
Fase estacionaria & Dimethyl posiloxane & Polietilenglicol en fase polar \\
Cantidad de muestra & $1,5 \mathrm{mg}+-1$ & $10 \mu \mathrm{L}$ \\
Tipo de inyección. & $\mathrm{Headspace}$ & Split o división. \\
Temperatura operación & $105^{\circ} \mathrm{C}$ & $250^{\circ} \mathrm{C}$ \\
Presión de operación & $3447.37 \mathrm{kPa}$ & $77 \mathrm{kPa}$ \\
\hline
\end{tabular}

a Columna, composición: $5(5 \%$ diphenyl) dimethyl posiloxane; diámetro de la columna: $0.25 \mathrm{~mm}$; longitud: $60 \mathrm{~m}$. ${ }^{\text {b }}$ Diámetro de la columna: 0,22 m; longitud de la columna: $50 \mathrm{~m}$.

\section{Prueba de destilación}

Se realizó una prueba de destilación por duplicado, conforme a lo establecido por la NTC 619 (Icontec, 1995). $100 \mathrm{~mL}$ de muestra fueron destilados empleado un montaje de destilación simple (ver figura 2), a presión atmosférica en Bogotá $(560 \mathrm{mmHg})$, bajo condiciones equivalentes a las empleadas para la destilación diferencial de un lote; posteriormente, se tomaron 9 fracciones del destilado, de $10 \mathrm{~mL}$ cada una, correspondientes a los diferentes porcentajes en volumen de solvente recuperado. Se midieron las temperaturas del vapor para cada fracción usando un termómetro de mercurio; cada una de las 9 fracciones de la prueba de destilación fue unida a sus respectivas réplicas para la obtención de 9 muestras compuestas, que fueron analizadas mediante cromatografía de gases. Las condiciones de operación de la cromatográfia de gases (CG) se presentan en la tabla 1.

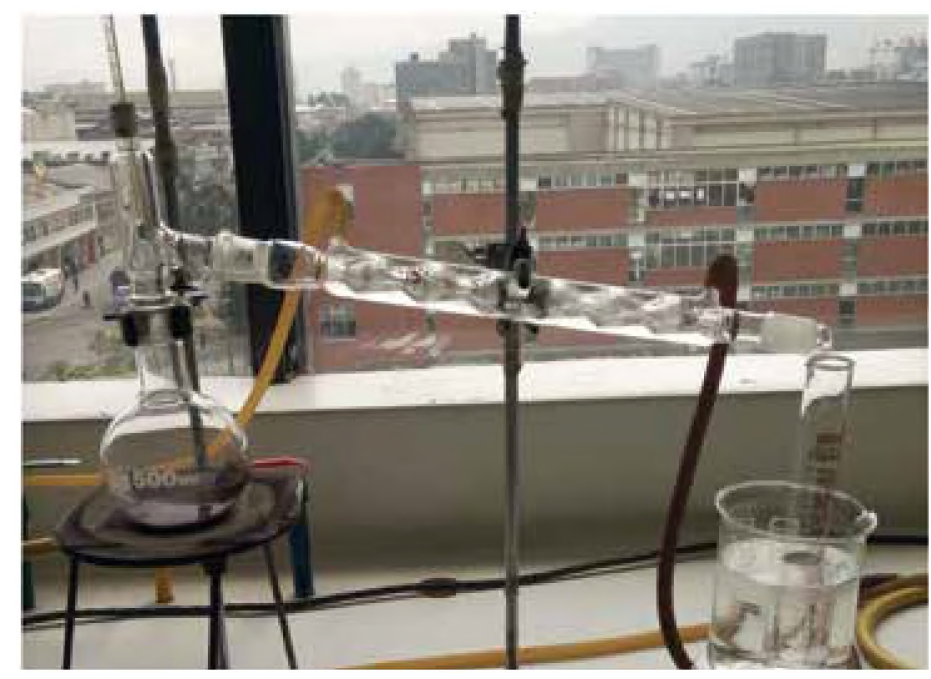

Figura 2. Montaje de destilación simple usado para la prueba de destilación. 
Las cantidades remanentes en los fondos o residuos de destilación, también conocidos como lodos de tinta, fueron cuantificadas gravimétricamente. Su caracterización cualitativa y posibilidades de reutilización serán objeto de futuras investigaciones.

Se realizó una gráfica de las temperaturas medidas en función del porcentaje de solvente recuperado. Adicionalmente y usando una modificación del modelo de una destilación diferencial propuesto por Bonsfills y Puigjaner (2004), se realizaron los balances de materia por diferencias finitas, con el fin de determinar los contenidos de los fondos correspondientes a cada fracción obtenida durante la prueba de destilación. Debido a la complejidad del sistema, que tiene diferentes compuestos orgánicos volátiles, sólidos solubles (nitrocelulosa) y sólidos no solubles (pirgmentos), se decidió realizar un primer acercamiento al problema con el análisis de las fracciones libres de sólidos presentes en los fondos de destilación. En la figura 3 se muestra una representación esquemática de la destilación diferencial.

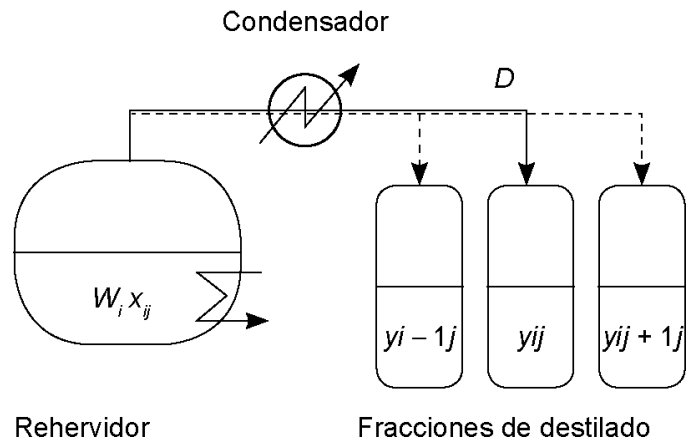

Figura 3. Representación esquemática de una destilación diferencial, con recolección de fracciones.

$\mathrm{Al}$ realizar los balances globales y por componente en el sistema de destilación simple, se obtienen las siguientes expresiones:

$$
\begin{gathered}
\frac{d M}{d t}=-D \\
\frac{d(M x j)}{d t}=-D y_{j}
\end{gathered}
$$

Donde $M$ corresponde a la cantidad de masa en el rehervidor en cualquier momento de tiempo; $x_{j}$ es la fracción másica libre de sólidos del componente $j$ en el líquido contenido en el rehervidor para cualquier momento de tiempo; $D$ representa el flujo másico del destilado, y $y_{j}$ es la fracción másica del componente $j$ en la corriente de vapor y, por lo tanto, la fracción másica en el destilado.

Combinando las ecuaciones 1 y 2 se obtiene:

$$
\frac{d\left(M x_{j}\right)}{d t}=\frac{d M}{d t} y_{j}
$$

Para un lapso $\Delta t$, correspondiente al tiempo de recolección de cada una de las nueve fracciones, con $i$ variando de 1 a 9 , se puede aplicar una diferencia finita al modelo para obtener: 


$$
\frac{\Delta\left(M x_{j}\right)_{i}}{\Delta t_{i}}=\frac{\Delta M_{i}}{\Delta t_{i}} y_{i j}
$$

Eliminando la fracción de tiempo, $\Delta t_{i}$, de la ecuación, se logra la siguiente expresión:

$$
\Delta\left(M x_{j}\right)_{i}=\Delta M_{i} y_{i j}
$$

Donde $\Delta M_{i}$ corresponde al cambio de masa en el rehervidor y por lo tanto a la masa recolectada en cada una de las fracciones, mientras que $y_{i j}$ es la fracción másica de cada una de las nueve fracciones en el destilado (valores conocidos mediante las cromatografías de gases). Por definición se tiene que:

$$
\Delta\left(M x_{j}\right)_{i}=\left(M x_{j}\right)_{i-1}\left(M x_{j}\right)_{i}=\Delta M_{i} y_{i j}
$$

Así que conociendo las cantidades iniciales de cada uno de los componentes en la muestra (calculadas como fracciones libres de sólidos), es posible realizar el seguimiento de la composición del contenido en el rehervidor (residuo) durante el proceso de destilación diferencial con las siguientes expresiones:

$$
\begin{gathered}
\left(M x_{j}\right)_{i}=\left(M x_{j}\right)_{i-1}=\Delta M_{i} y_{i j} \\
x_{i j}=\frac{\left(M x_{j}\right)_{i-1}-\Delta M_{i} y_{i j}}{M_{i}} ; M_{i}=M_{i-1}-\Delta M_{i}
\end{gathered}
$$

Una vez calculadas las fracciones de cada componente volátil en el rehervidor, los datos son graficados para analizar el desarrollo de la prueba de destilación.

\section{Simulación con Aspen Plus}

Posteriormente, conociendo la presión del sistema, la naturaleza de las sustancias involucradas, la disponibilidad de parámetros y el tipo de equilibrio, se empleó el árbol de decisión propuesto por Carlson (1996), con el que se determinó que los modelos termodinámicos NRTL y UNIQUAC son los más pertinentes para realizar una simulación en el programa Aspen Plus. Con este programa se calcularon los puntos de burbuja correspondientes a cada conjunto de composiciones $x_{i j}$ en la fase líquida del rehervidor, al igual que las composiciones en la fase vapor $y_{i j}$, que estarían en equilibrio con dichas composiciones de la fase líquida. Estos resultados fueron comparados con las temperaturas halladas experimentalmente durante la prueba de destilación y las composiciones de la fase vapor obtenidas por cromatografías de gases de las facciones de solvente recuperadas.

Se realizaron los mapas de residual para los modelos termodinámicos NRTL y UNIQUAC con el simulador Aspen Plus, empleando las composiciones normalizadas de los tres componentes mayoritarios (1-propanol, etanol y acetato N-propilo) con los datos de fracción molar en la fase 
líquida, determinados con el método de diferencias finitas, a partir de los que obtuvo una representación gráfica del proceso de destilación. (Sánchez-Daza, Vidriales, Morales y Ortiz, 2006)

\section{Resultados Y DISCUSIÓN}

\section{Caracterización del residuo de tinta}

Mediante el método de Karl Fischer se determinó que los residuos de tinta tenían una humedad de $2.1680 \pm 0.057 \%$, mientras que el material no volátil fue $4.7573 \pm 0.0341 \%$. Teniendo en cuenta que las fichas técnicas de los solventes e insumos usados por la empresa indican contenidos de humedad inferiores, se puede inferir que la humedad de las muestras proviene principalmente de procesos de absorción desde el medio ambiente hacia la masa de residuos de tinta; sin embargo, para estas muestras, el agua permanece como un constituyente minoritario dentro del residuo. También se sabe que la fracción sólida no volátil de estos residuos está compuesta por resinas de nitrocelulosa y pigmentos procedentes de los insumos de impresión (Izdebska y Thomas, 2016), cuya mezcla es responsable del color violáceo oscuro de los residuos (una evaluación más rigurosa de su composición será objeto de futuras investigaciones).

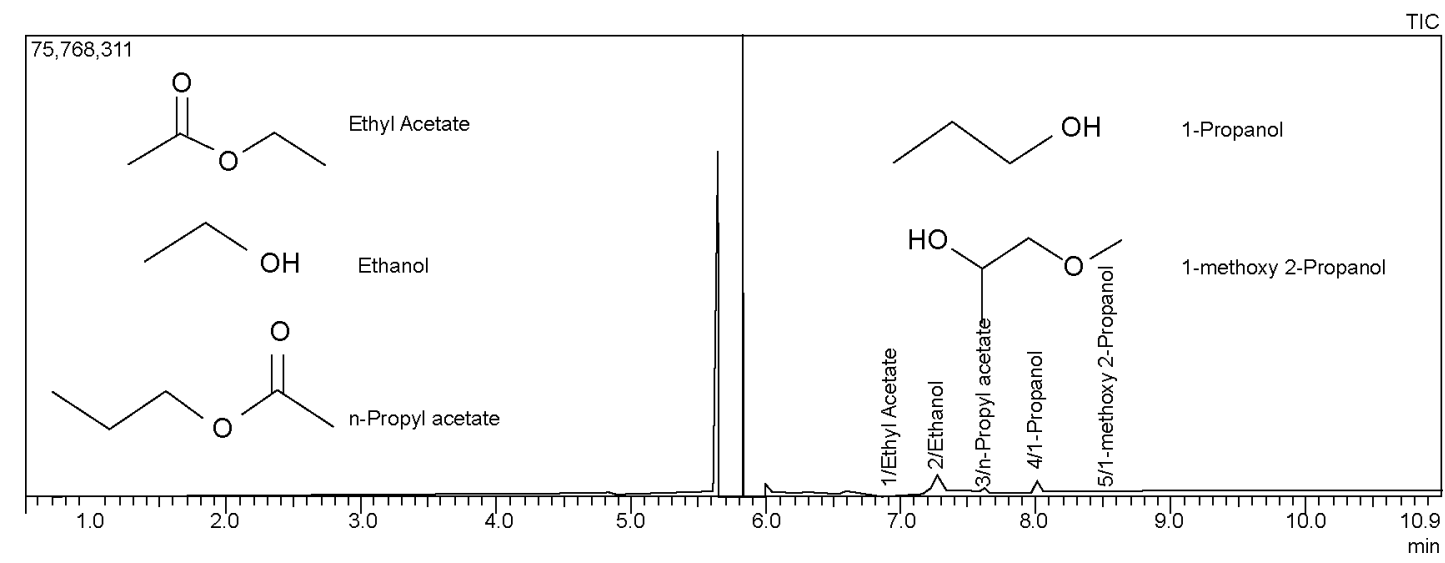

Figura 4. Resultados de la cromatografía de gases acoplada a espectro de masas de la muestra de residuo de tinta.

En la figura 4 se presentan los resultados de la cromatografía de gases acoplada a espectro de masas, en la que se determinó la presencia de cinco compuestos, con picos bien definidos en diferentes tiempos de retención (desde 6.887 min hasta $8.504 \mathrm{~min}$ ). En la tabla 2 se presentan las composiciones de los compuestos en la muestra estudiada.

Tabla 2. Composición de la fracción volátil del residuo de tinta

\begin{tabular}{lccc}
\hline \multicolumn{1}{c}{ Sustancia } & Peso molecular $\mathbf{~ g / m o l ~}$ & Concentración en masa (\%) & Concentración molar (\%) \\
\hline Propanol & 60.10 & 60.69 & 64.75 \\
Etanol & 46.07 & 13.59 & 18.91 \\
N-propil acetato & 102.13 & 14.85 & 9.32 \\
Acetato de etilo & 88.11 & 7.29 & 5.30 \\
Metoxipropanol & 90.12 & 2.41 & 1.71 \\
Desconocidos & & 1.17 & N.A. \\
\hline
\end{tabular}

${ }^{a}$ La concentración molar se expresa como libre del material desconocido. 
Se puede observar que el componente mayoritario es el etanol, seguido por el propanol y el Npropil acetato, con trazas de acetato de etilo y metoxipropanol. Los compuestos encontrados son consistentes con los contenidos en las materias primas usadas para la formulación de las tintas y en los solventes usados para las operaciones de limpieza de los equipos de impresión.

En la figura 5 se muestra un resumen de la composición de los residuos de tinta, expresado en porcentajes de la masa total del residuo.

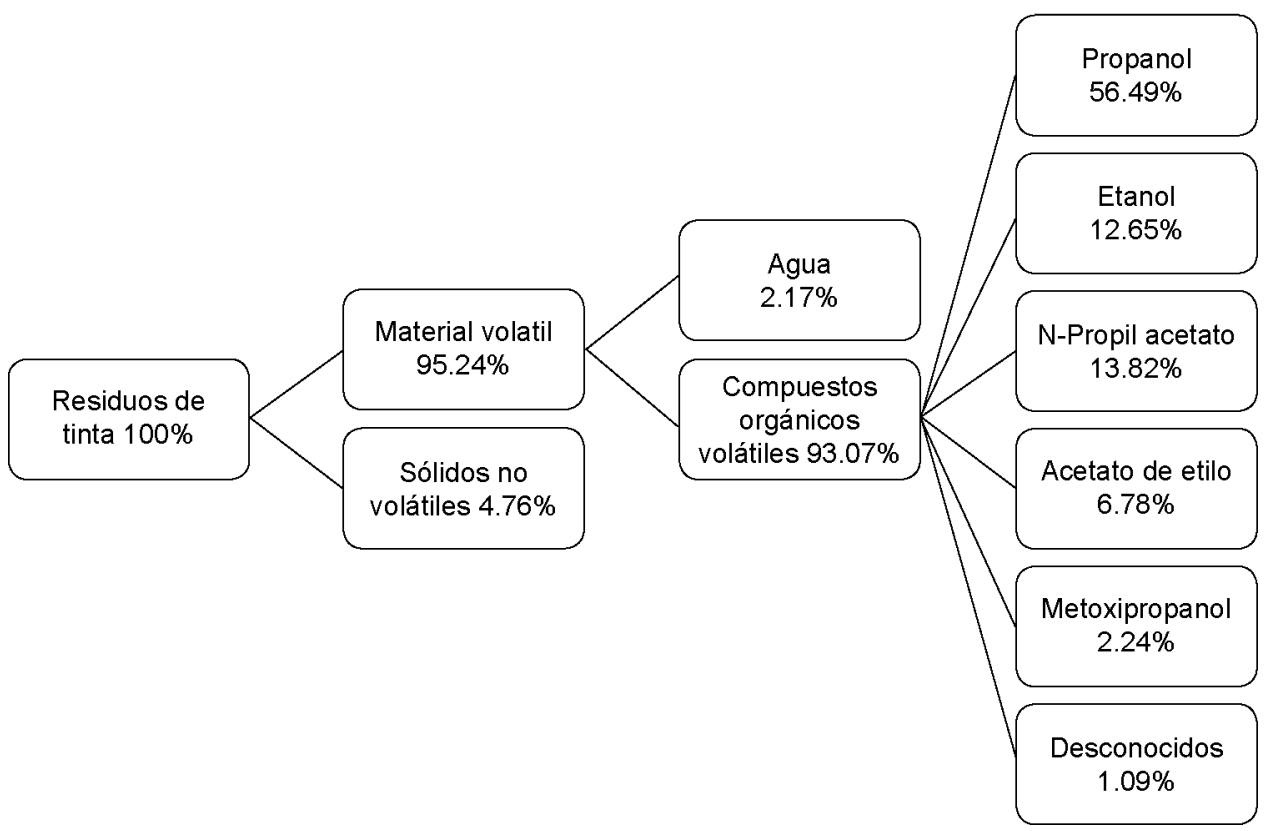

Figura 5. Composición de los residuos de tinta.

\section{Prueba de destilación}

Después de realizar la prueba de destilación, cada una de las 9 fracciones obtenidas fue unida a su réplica para ser analizadas mediante cromatografía de gases. En la figura 2 se presenta el resultado de la cromatografía para la fracción 1, correspondiente al $11 \%$ del volumen de solvente recuperado. En la figura se observan los picos con sus respectivos tiempos de retención.

En la tabla 3 se presentan las composiciones de las nueve fracciones recogidas durante la prueba de destilación. Se puede observar que el componente mayoritario de todas las fracciones es el propanol, seguido por el propanol y el $\mathrm{N}$ propil acetato.

Los tres constituyentes principales, propanol, etanol y N-Propil acetato, aportan en conjunto entre el 84 y el $93 \%$ del contenido total en todas las fracciones, mientras que los constituyentes minoritarios, acetato de etilo y metoxipropanol, aportan entre el 15.81 y el $6.52 \%$. Los componentes desconocidos están por debajo del $2.03 \%$ para todas las muestras.

Teniendo en cuenta que, para lograr las condiciones de estabilidad y viscosidad requeridas para un buen desempeño sobre el sustrato la formulación de la tinta requiere, para el clima de Bogotá, de una composición cercana al 75\% de alcoholes y 25\% de ésteres de ácido acético (Guerrero, Tintas en la industria flexográfica, 2017), se establece que la recolección de las cuatro últimas fracciones puede proporcionar una parte del solvente a usar para tal fin ya que su composición acumulativa corresponde a un $74.96 \%$ de alcoholes (propanol y etano) y $24.24 \%$ de ésteres de ácido acético (npropil acetato y acetato de etilo), representando el $44 \%$ del solvente recuperado; el $56 \%$ del solvente 
restante, correspondiente a las cinco fracciones iniciales posee una composición del $72.55 \%$ de alcoholes y $26.32 \%$ de ésteres de ácido acético, pudiendo se usar como parte de la formulación para las operaciones de la limpieza de las unidades de impresión.

Aplicando los balances de masa mediante el modelo de diferencias finitas propuesto, se obtuvieron las composiciones en rehervidor durante la prueba de destilación, resultados que se presentan en la figura 7, en ella se aprecia un comportamiento de aumento para el propanol, y el metoxipropanol, el componente menos volátil de la mezcla, mientras que el resto de constituyentes poseen una tendencia a decrecer durante la destilación, en especial el acetato de etilo, que es el componente más volátil.

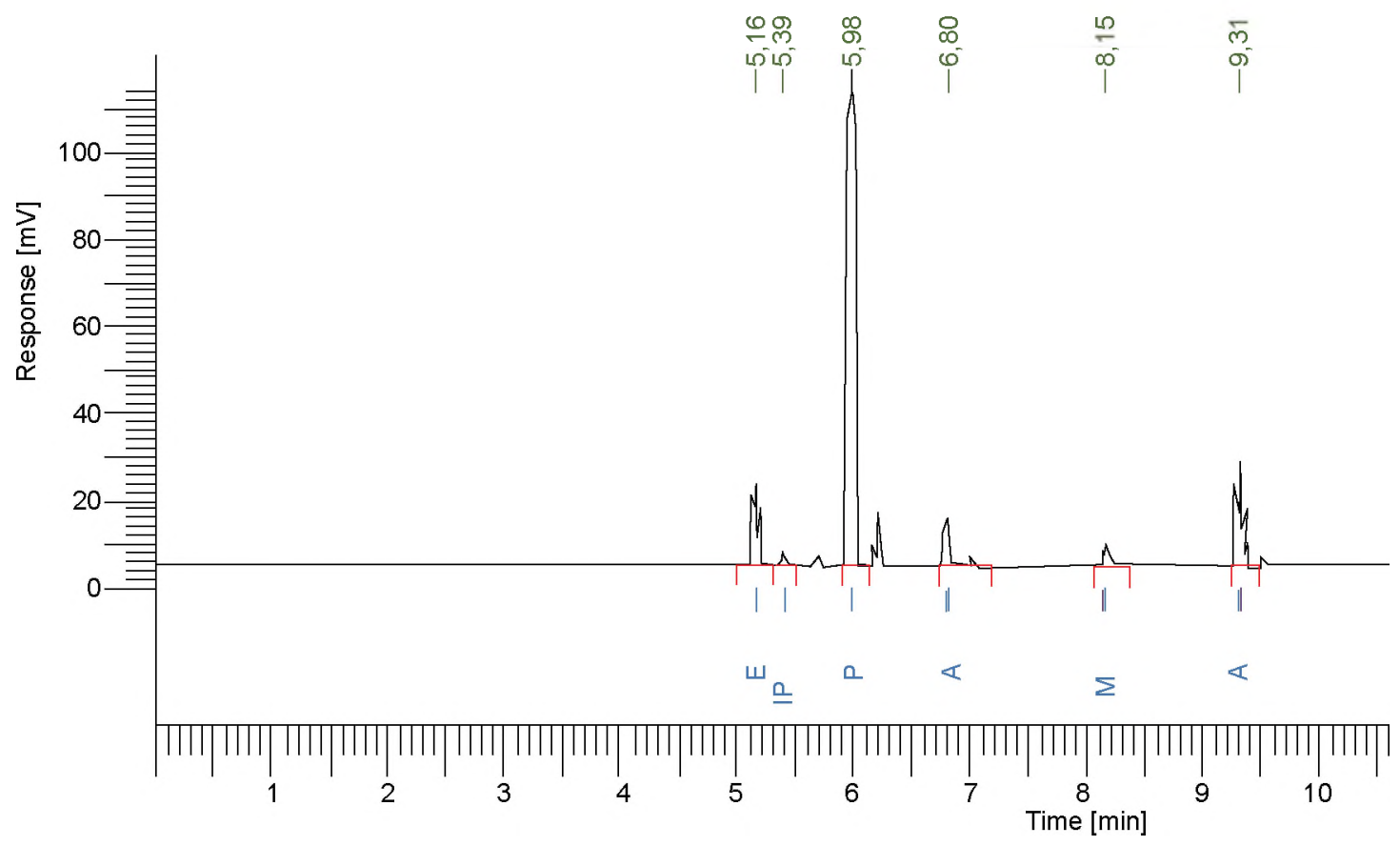

Figura 6. Resultado de la cromatografía de gases para la fracción 1. En orden de tiempos de retención, de izquierda a derecha: etanol $(E)$, propanol $(P)$, acetato de etilo $(A)$ metoxipropanol $(M)$

y $\mathrm{N}$-Propil acetato $(\mathrm{A})$.

Tabla 3. Porcentajes másicos de los compuestos orgánicos volátiles en las fracciones obtenidas durante la prueba de destilación

\begin{tabular}{lccccccccc}
\hline \multicolumn{1}{c}{ Fracción (i) } & $\mathbf{1}$ & $\mathbf{2}$ & $\mathbf{3}$ & $\mathbf{4}$ & $\mathbf{5}$ & $\mathbf{6}$ & $\mathbf{7}$ & $\mathbf{8}$ & $\mathbf{9}$ \\
\hline Volumen recuperado (\%) & 11.1 & 22.2 & 33.3 & 44.4 & 55.6 & 66.7 & 77.8 & 88.9 & 100.0 \\
Volumen recuperado (mL) & 10 & 20 & 30 & 40 & 50 & 60 & 70 & 80 & 90 \\
\hline Propanol & 61.34 & 45.69 & 54.24 & 55.13 & 59.91 & 61.30 & 66.08 & 69.88 & 73.77 \\
Etanol & 12.60 & 19.05 & 16.47 & 14.72 & 16.45 & 14.74 & 12.91 & 10.14 & 7.95 \\
N-propil acetato & 12.70 & 19.43 & 17.75 & 14.61 & 16.72 & 14.94 & 14.07 & 12.49 & 10.70 \\
Acetato de etilo & 8.23 & 15.81 & 9.51 & 13.43 & 6.90 & 5.70 & 4.35 & 2.87 & 1.75 \\
Metoxipropanol & 3.37 & 0.00 & 0.00 & 2.11 & 0.00 & 1.95 & 2.59 & 3.65 & 4.91 \\
Desconocidos & 1.75 & 0.02 & 2.03 & 0.00 & 0.02 & 1.37 & 0.00 & 0.97 & 0.92 \\
\hline
\end{tabular}




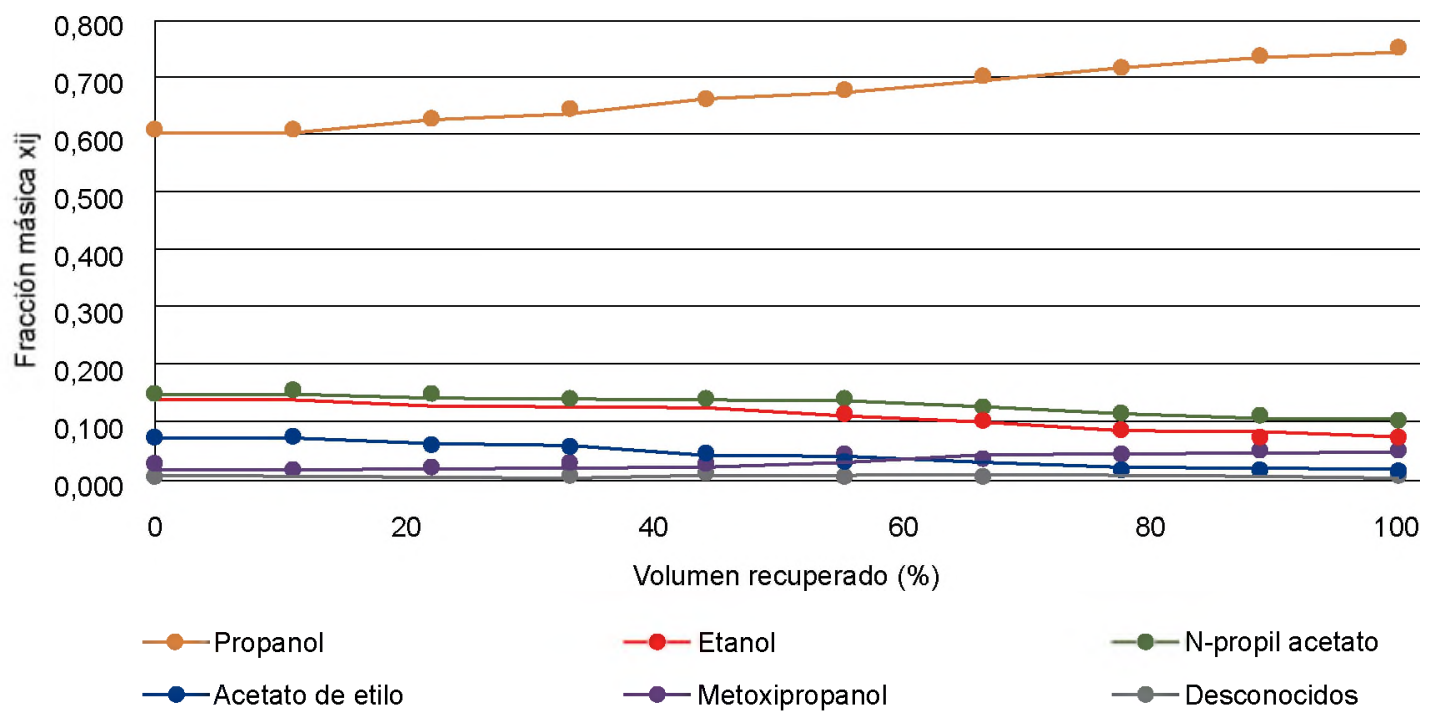

Figura 7. Evolución de la composición en el rehervidor durante la prueba de destilación.

Se encontró que un $10.616 \pm 0.294 \%$ de los residuos de tinta se convirtieron en lodos de tinta. Estos lodos están compuestos de un $44.81 \%$ de sólidos, una humead del $7.46 \%$ y un contenido en diversos compuestos orgánicos volátiles del $47.73 \%$. Otros autores han reportado concentraciones de sólidos entre el 35 (Pineda et ál., 2011) y el 75 \% (Yan et ál., 2013), lo que era de esperarse, ya que la composición de dichos lodos depende del proceso de recuperación de solventes al que haya sido sometido el residuo de tinta, y no solo de la naturaleza del residuo; razón por la cual la estandarización del método de recuperación de solventes proveerá de un lodo de tinta, también estandarizado, que facilitaría las operaciones de recuperación de sus componentes.

\section{Simulación con Aspen Plus}

En la figura 8 se presenta la evolución de la temperatura para cada uno de los puntos como un promedio de las dos determinaciones experimentales y la predicción de los puntos de burbuja. Aquí se usó el programa Aspen Plus, con los modelos termodinámicos UNIQUAC y NRTL y la base de datos de propiedades y parámetros disponibles para el programa.

Se puede observar una importante diferencia entre los valores experimentales y los calculados para los puntos de burbuja; aunque para este trabajo no se determinó la composición de la fracción sólida. Se sabe que los pigmentos y aditivos de las tintas tienen resinas a base de nitrocelulosa y algunas sales inorgánicas de elevado peso molecular, al igual que material insoluble que forma emulsiones con los solventes (Izdebska y Thomas, 2016). Los iones de las sales tienen la predisposición a acumularse con moléculas más polares y formar complejos que alteran la presión de vapor de los constituyentes de la mezcla (Ohe, 1998). Teniendo en cuenta que los residuos de tinta tienen en un inicio un contenido de sólidos de $4.76 \%$, habría que esperar un incremento del punto de burbuja de la mezcla por encima del de la mezcla libre de sólidos (Levine, 2004); sin embargo, para el $78 \%$ inicial del volumen recuperado, los datos experimentales muestran lo contrario frente a lo calculado con los dos modelos termodinámicos. Esto señala que los parámetros empleados por el programa requieren de ajustes para predecir adecuadamente las temperaturas de la mezcla trabajada bajo las condiciones de operación de la prueba. 


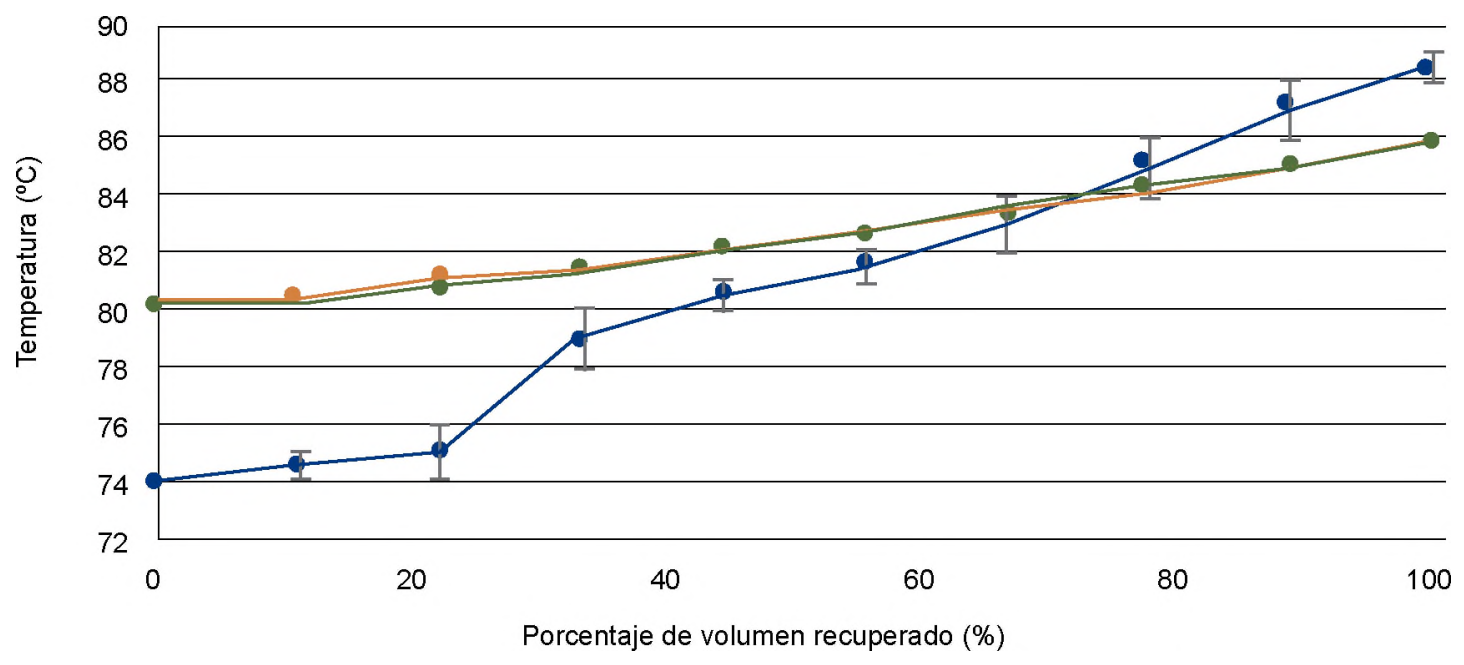

$\longrightarrow$ Promedio

$\longrightarrow$ Modelo Uniquac T Burbuja

$\longrightarrow$ Modelo NRTL T Burbuja

Figura 8. Curva de destilación, comparación con modelos NRTL y UNIQUAC.

A medida que la avanza la destilación, los componentes más volátiles abandonan la mezcla, concentrándose los componentes menos volátiles, lo que aumenta la temperatura del punto de burbuja. Los dos modelos predicen dichos incrementos en forma similar, lo que se evidencia en la pendiente de sus gráficas. Al mismo tiempo, la concentración de sólidos en la mezcla se va incrementando desde un $4.76 \%$, al inicio de la destilación, hasta alcanzar el $47.73 \%$, cuando esta finaliza, lo que causa un aumento mayor de dicha temperatura por efecto de las propiedades coligativas (Ohe, 1998). Esto se ilustra en una pendiente mayor en la gráfica de los datos experimentales, frente a la mostrada por los dos modelos termodinámicos, lo que hace que a partir del $78 \%$ del volumen recuperado la temperatura experimental esté sobre la temperatura predicha por dichos modelos.

Por otra parte, son pocos los estudios que han tratado mezclas con más de dos alcoholes, en estos casos los enlaces de hidrógeno se forman como asociaciones de especies, por lo cual, el álgebra involucrada para llegar a un modelo de solución es complejo, según (A.G. Pradhan,1993), el efecto de las asociaciones en el modelo UNIQUAC es más empleado para mezclas binarias y ternarias que abarcan generalmente dos alcoholes, siendo este modelo insuficiente para determinar efectos en las interacciones físicas o ramificaciones que pueden ser importantes. Sin embargo, éste permite estimaciones razonables para equilibrio líquidovapor respecto a sistemas binarios y ternarios que involucran alcoholes, ésteres y cetonas; mientras que el modelo NRTL, es conveniente para representar mezclas multicomponente que contienen alcohol basado en estudios de simulación que se han realizado para este modelo (Wagner L.R., 2013).

En la figura 9 se muestran los mapas de curva residual con los modelos NRTL y UNIQUAC. Las curvas intermedias indican la presencia de dos azeótropos desde el nodo inestable hasta el punto de silla; por su parte, la sucesión de círculos azules en la parte inferior derecha representa las composiciones calculadas en el rehervidor y, por lo tanto, la línea de operación de la destilación diferencial realizada. El punto 1 corresponde a la alimentación, mientras que el punto 9 representa la composición de estos tres elementos volátiles en el lodo residual. 


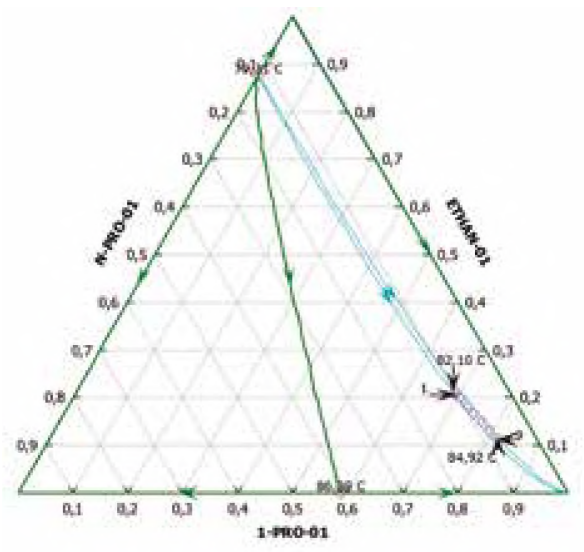

(A)

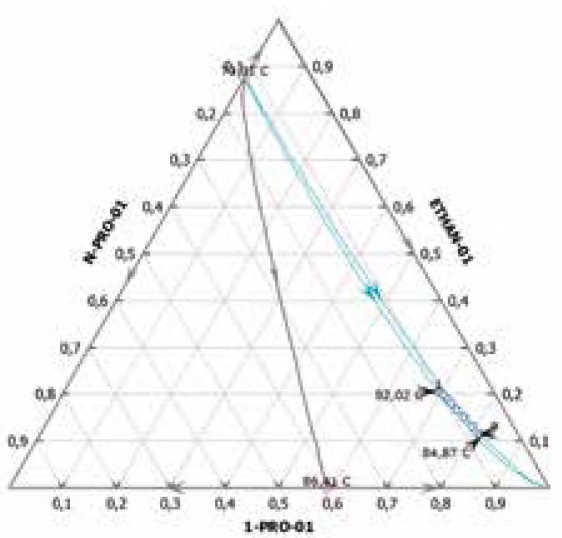

(B)

Figura 9. Mapas de curvas residuales: a) modelo UNIQUAC; b) modelo NRTL

Las líneas azules corresponden a la composición de residuos calculada por el programa para las composiciones normalizadas de los tres compuestos orgánicos volátiles mayoritarios, tanto para el alimento (línea que pasa por el punto 1), como para el residuo de lodos de fondo (línea que pasa por el punto 2). Se observa en estas curvas una tendencia hacia el aumento de la temperatura, que se concentra hacia el propanol. La cercanía de las dos líneas de residuos predichas por el programa muestra una buena correlación de las composiciones de fondos frente a los datos experimentales. Es de esperar que con los ajustes adecuados se logre una mejor predicción de los puntos de burbuja de la mezcla y de las curvas de residuos; información con la que se podría diseñar y simular la operación del equipo de destilación para la recuperación de los solventes como una mezcla, e incluso para la separación de los componentes mayoritarios como sustancias puras.

\section{Conclusiones}

Se evidencia que existe un proceso de absorción de humedad hacia los solventes recuperados desde el aire, ya que la humedad encontrada en los residuos no proviene de las materias primas ni de los solventes usados durante las operaciones de impresión o lavado de los equipos, sin embargo, el agua permanece como un compuesto minoritario en el residuo.

Los componentes mayoritarios en los residuos de tinta son el propanol, el etanol y el n-propil acetato, lo que es consistente con los contenidos en las materias primas usadas para la formulación de las tintas y la limpieza de los equipos; el residuo de tinta posee una composición del $4.76 \%$ en sólidos no volátiles, que de acuerdo con las fichas técnicas de las tintas corresponden a las resinas y pigmentos usados en la impresión.

La metodología presentada en la NTC 319 puede ser potencializada con el uso de cromatografías de gases, modelos del proceso de destilación y empleo programas de simulación, para generar herramientas que permitan el diseño y operación de sistemas de destilación ajustados a las necesidades del sector de impresión, el que requiere de mezclas específicas de solventes para sus operaciones de formulación de tintas y lavado de equipos, sin la necesidad de separar los constituyentes del residuo en sus componentes puros.

Se evidencia un aumento de la temperatura durante la operación de destilación, la que se puede explicar por la disminución de los componentes volátiles en la mezcla a medida que va aumentando el volumen de solvente recuperado, por otro lado, la presencia de sólidos solubles (nitrocelulosa) y no solubles (pigmentos), en la mezcla estudiada genera un incremento adicional en 
las temperaturas durante el desarrollo de la operación de destilación debido al efecto incremental de la concentración de dichos sólidos en el rehervidor.

Aunque los resultados obtenidos por los dos modelos termodinámicos NRTL y UNIQUAC son consistentes entre sí, no logran predecir adecuadamente las temperaturas experimentales medidas durante la prueba de destilación, las que inician en valores inferiores a las estimadas por los modelos incrementándose hasta valores superiores a los predichos por los mismos al final de la operación de destilación, esto se debe a que en este estudio no se evaluó el efecto que posee el incremento en la concentración de los sólidos solubles (resinas) y no solubles (pigmentos), sobre la temperatura de burbuja.

Para este caso, el principal objetivo de la operación de destilación de residuos líquidos es la separación de los solventes de las sustancias pesadas, se encontró que una destilación simple sin rectificación sería suficiente para lograr dicho objetivo, pudiéndose obtener dos fracciones de mezcla de solventes, la primera correspondiente al 56\% del solvente recuperado la que posee unas proporciones cercanas a $78-82 \%$ de alcoholes y $18-22 \%$ de éster de ácido acético por lo que es adecuada para las operaciones de limpieza de los equipos de impresión (Guerrero, Tintas en la industria flexográfica, 2017) , mientras que la segunda, correspondiente al $44 \%$ restante, puede ser usada para la formulación de las tintas.

\section{ReCOMENDACIONES}

A pesar de que los modelos termodinámicos estudiados no predicen adecuadamente los puntos de burbuja de la mezcla destilada, los diagramas de residuos elaborados con las composiciones de los tres compuestos orgánicos volátiles mayoritarios, proveen de una adecuada predicción del comportamiento de la composición del rehervidor durante la destilación diferencial, se recomienda realizar ajustes al modelo, incluyendo el efecto de los sólidos y su incremento durante la destilación, al igual que el ajuste de los parámetros, con el fin de lograr mejores predicciones de las temperaturas del proceso y así poder emplear el mismo para el diseño y operación del equipo de destilación.

Se recomienda evaluar opciones tecnológicas para la recuperación de los pigmentos y resinas contenidas en el lodo residual, producto de fondos de la operación de destilación, toda vez que en la actualidad éstos son dispuestos en celdas dentro de los rellenos sanitarios o incluidos como material de carga durante el proceso de fabricación de cemento, desperdiciando un material valioso y contribuyendo a el aumento de la contaminación.

\section{Referencias}

Andigraf. (2014). Informe de sostenibilidad 2013 Industia editorial y de la comunicación gráfica. Bogotá D. C.: Andigraf. Recuperado de http://www.andigraf.com.co/files/informe_economico/2015/informe_de_sostenibilidad_2013_industria_editorial_y_de_la_comunicacion_grafica.pdf

Andigraf. (2017). Informe de las Empresas de la Industria gräfica en Colombia. Bogotá D. C.: Andigraf. Recuperado de http:/www.andigraf.com.co/informes-economicos

Bonsfills, A., y Puigjaner, L. (2004). Batch distillation: simulation and experimental validation. Chemical Engineering and Processing, 43(10), 1239-1252. doi:10.1016/j.cep.2003.11.009

Carlson, E. (1996). Don't gamble with physical properties for simulations. Chemical Engineering Progress, 92(10), 36-46. 
Fullana, A., Aracid, I., y Pineda, A. (2011). Recuperación de tinta a partir de residuos de la industria gráfica. Ingenieria Quimica, 492, 68-70.

Icontec. (1974). Pinturas. Disolventes. Determinación de la materia no volátil (NTC 762). Bogotá D. C.: Icontec.

Icontec. (1995). Método de ensayo para el intervalo de destilación de líquidos orgánicos volátiles (NTC 319). Bogotá D. C.: Icontec.

Icontec. (2008). Pinturas, barnices y materias primas. Muestreo (NTC 1052). Bogotá D. C.: Icontec.

IDEAM. (2016). Informe nacional generación y manejo de residuos o desechos peligrosos en Colombia, 2014. Bogotá D. C.: IDEAM. Recuperado de http://www.andi.com.co/Ambiental/Documents/Informe\%20Nacional\%20de\%20Residuos\%20Peligrosos\%20\%202014\%202015.pdf

Izdebska, J., y Thomas, S. (2016). Printing on polymers Fundamentals and Applications. Oxford, Reino Unido: Elsevier. doi:10.1016/B978-0-323-37468-2.00001-4

Levine, I. (2004). Fisicoquimica (5ª edición). Aravaca, España: McGraw-Hill.

Ministerio de Industria y Turismo. (2017). Dinámica de la economía mundial y comportamiento en Colombia. Primer trimestre de 2017. Bogotá D. C.: Ministerio de Industria y Turismo. Recuperado de http://www.mincit.gov.co

Ohe, S. (1998). Prediction of salt effect on vapor-liquid equilibria. Fluid Phase Equilibria, 144(1), 119-129.

Pradhan, A., Bhethanabotla, V., y Campbell, S. (1993). Vapor-liquid equilibrium data for ethanol-n-heptane-I-propanol and ethanol-n -heptane-2-propanol and their interpretation by a simple association model. Fluid Phase Equilibria, 84, 183-206. doi:10.1016/03783812(93)85123-4

Rivera, J., y Pérez, M. (2006). Empleo del sistema potenciométrico Karl Fisher en el desarrollo y la certificación de materiales de referencia. Recuperado de https://www.cenam.mx/memsimp06/Trabajos\%20Aceptados\%20para\%20CD/Octubre\%2025/Bloque\%20A/A2-QUIMICA\%20I-Materiales\%20de\%20Referencia/A2-3.pdf

Sánchez-Daza, O., Vidriales, G., Morales, y Ortiz, E. (2016). Reactive residue curve maps: A new study case. Chemical Engineering Journal, 117(2), 123-129.

Smallwood, I. (2002). Solvent Recovery Handbook, Second edition. Oxford, Reino Unido: Blackwell Science Ltd.

Sorensen, E. (2014). Design and Operation of Batch Distillation. En A. Górak (ed.), Distillation: Fundamentals and Principles (pp. 187-224). London: Elsevier. doi:10.1016/B978-0-12386547-2.00001-6

Souza, W., Silva, C., y Meleiro, L. (2013). Vapor-liquid equilibrium of the (water + ethanol + glycerol) system: Experimental and modelling data at normal pressure. The Journal of Chemical Thermodynamics, 67, 106-111. doi:10.1016/j.jct.2013.07.012

Robert, T. (2015). “Green ink in all colors" Printing ink from renewable resources. Progress in Organic Coatings, 78, 287-292.

Yan, F., Chen, G., Zhu, K., y Yao, R. (2013). Research on Recycling and Utilization of Waste Ink. Advanced Materials Research, 800,67-71. doi:10.4028/www.scientific.net/AMR.800.67 